\title{
Intraarticular Tramadol-Bupivacaine Combination Prolongs the Duration of Postoperative Analgesia After Outpatient Arthroscopic Knee Surgery
}

Ahed Zeidan, MD*

Rida Kassem, MD+

Nazih Nahleh, MD*

Hilal Maaliki, MD*

Mohamad El-khatib, PhD‡

Michel M.R.F. Struys, MD,

$\operatorname{PhD\S } \| \mathbb{I}$

Anis Baraka, MD, FRCA
BACKGROUND: Intraarticular (IA) local anesthetics are often used for the management and prevention of pain after arthroscopic knee surgery. Recently, IA tramadol was also used for the management of these patients. However, the IA combination of local anesthetic and tramadol has not been evaluated in arthroscopic outpatients. Our primary aim in this study was to evaluate the analgesic effect of an IA combination of bupivacaine and tramadol when compared with each drug alone using visual analog scale (VAS) pain scores in patients undergoing day-care arthroscopic knee surgery. Additionally, we assessed analgesic demand.

METHODS: Ninety ASA I/II patients undergoing arthroscopic partial meniscectomy, performed by a single surgeon under general anesthesia, were assigned in a randomized, double-blind manner into three groups: group B $(n=30)$ received $0.25 \%$ bupivacaine, group T $(n=30)$ received $100 \mathrm{mg}$ tramadol, and group BT $(n=$ 30) received $0.25 \%$ bupivacaine and $100 \mathrm{mg}$ tramadol to a total volume of $20 \mathrm{~mL}$ by the IA route after surgery. Postoperative pain scores were measured on a VAS, at rest and on mobilization at $0.5,1,2,4,6,8,12$, and $24 \mathrm{~h}$. Duration of analgesia, the subsequent $24 \mathrm{~h}$ consumption of rescue analgesia, time to ambulation, and time to discharge were evaluated. In addition, the systemic side effects of the IA injected drugs were also assessed.

RESULTS: The results showed significantly lower VAS pain scores in group BT ( $P \ll$ 0.1 ) when compared with groups $T$ and B. Group BT had a later onset of postsurgical pain and longer time to first rescue analgesic than groups B and T. The $24 \mathrm{~h}$ consumption of analgesic was significantly less in group BT when compared with the other two groups ( $26.7 \%$ of the patients required rescue analgesia in group $\mathrm{BT}$, whereas this number was $90 \%$ in group B and $86.7 \%$ in group T). In addition, time in hours to discharge and time to unassisted ambulation were significantly shorter in group BT when compared with groups $\mathrm{T}$ and B, and this was not associated with any detectable systemic effects.

CONCLUSION: The IA admixture of tramadol $100 \mathrm{mg}$ with bupivacaine $0.25 \%$ provides a pronounced prolongation of analgesia compared with either drug alone in patients undergoing day care arthroscopic knee surgery.

(Anesth Analg 2008;107:292-9)

A

rthroscopic knee surgery is commonly performed as an outpatient procedure and is often associated with postoperative pain. Intraarticular (IA) local anesthetics (LA) are often used for prevention of pain after arthroscopic knee surgery; however, the degree of postoperative pain varies. In an effort to find the ideal

From the Departments of *Anesthesiology, +Orthopedic surgery, Sahel General Hospital, and ‡Department of Anesthesiology, American University of Beirut Medical Center, Beirut, Lebanon; $\S$ Department of Anesthesia, Ghent University Hospital, |Department of Anesthesia, and IIHeymans Institute of Pharmacology, Ghent University, Gent, Belgium.

Accepted for publication January 22, 2008.

Address correspondence and reprint requests to Ahed Zeidan, Department of Anesthesiology, Sahel General Hospital, Airport Ave., P. O. BOX 99/25-Ghobeiry, Beirut, Lebanon. Address e-mail to doczeidan@hotmail.com.

Copyright (C) 2008 International Anesthesia Research Society DOl: 10.1213/ane.0b013e31816ba364 regime for sufficient, long-lasting postoperative analgesia, many different drugs, including opioids, nonsteroidal antiinflammatory drugs, ketamine, clonidine, and neostigmine, have been added to the IA LAs. ${ }^{1-4}$ The analgesic effect of IA tramadol after arthroscopic knee surgery has been reported recently by Alagol et al., ${ }^{5}$ who reported that IA tramadol $100 \mathrm{mg}$ without LA provided a longer alternative analgesic effect than after IV injection of the same dose of tramadol. However, the IA combination of LA and tramadol had not been evaluated in outpatients undergoing arthroscopic knee surgery.

The primary aim of this study was to compare the analgesic effect of IA bupivacaine $0.25 \%$ and tramadol $100 \mathrm{mg}$, used separately, and evaluate if their combination would provide superior analgesia to each drug alone, as assessed by the Visual Analog pain Scores at rest (VASr) and on mobilization (VASm). The secondary end-points were duration of analgesia, as defined 
by first demand for analgesia ( $1 \mathrm{~g}$ of oral paracetamol), and subsequent $24-h$ consumption. The time of unassisted ambulation, time to discharge, and side effects were also assessed.

\section{METHODS}

After IRB approval and informed written consents, 90 unpremedicated patients scheduled to undergo elective arthroscopic surgery by a single surgeon were included in this prospective, randomized, doubleblind study. Patients eligible for participation were older than $18 \mathrm{yr}$, and were ASA physical status I or II. Patients excluded were those treated with narcotics preoperatively and those who had a contraindication to the use of bupivacaine or tramadol.

Before the operation, all patients received instructions for using a $100-\mathrm{mm}$ VAS score (with $0=$ no pain, to $100=$ the worst imaginable pain). The baseline pain scores were recorded postoperatively. Pain was assessed by a single interviewer who was not aware of the study medication. Anesthesia was induced with propofol $(2.5 \mathrm{mg} / \mathrm{kg})$, rocuronium $(0.6 \mathrm{mg} / \mathrm{kg})$ and fentanyl $(0.002 \mathrm{mg} / \mathrm{kg})$ and maintained with nitrous oxide $60 \%$ in oxygen and sevoflurane. No other supplementary analgesic medication was given during the operation after the first dose of fentanyl. During anesthesia, controlled ventilation was performed via an endotracheal tube. Before surgical incision, a thigh pneumatic tourniquet on the same side as the surgery, at a pressure of $300 \mathrm{~mm} \mathrm{Hg}$, was applied to all patients. The same surgeon performed all surgical procedures using a standard surgical technique.

At the end of the operation, patients were allocated, using a randomized number table, into 1 of 3 groups, consisting of 30 patients each. Group T received 100 mg of IA tramadol, group B received $0.25 \%$ bupivacaine, and group BT received a mixture of $0.25 \%$ bupivacaine and tramadol $100 \mathrm{mg}$. The volume of the injectate was standardized at $20 \mathrm{~mL}$. The study solution, supplied in a coded syringe, was injected by the surgeon into the knee joint through an arthroscope at the end of surgery, $10 \mathrm{~min}$ before tourniquet release.

After the end of anesthesia, patients were transferred to the postanesthesia care unit. Arrival at the postanesthesia care unit was recorded as time zero. The VAS was assessed at predetermined intervals after surgery $(0.5,1,2,4,6,8,12$, and $24 \mathrm{~h})$. At each time of measurement, pain scoring was performed at rest (VASr) and on mobilization (VASm) (bending of the operated knee). When patients complained of pain (VAS score more than 40), they were given $1 \mathrm{~g}$ of paracetamol orally as a rescue medication. Duration of effective analgesia was measured from the time of surgery completion until first requirement of rescue analgesia.

When patients were discharged, they were given a data sheet and they were instructed how to evaluate the degree of pain by using the VAS score ruler.
Therefore, they could read by themselves the corresponding numerical score, record it on the data sheet at the predetermined times, and report their analgesic consumption. Also, the patients were asked to record any adverse effect, such as headache, dizziness, somnolence, nausea, and vomiting.

Patients were discharged from the hospital when they were oriented to time and place, were able to void, had stable vital signs, and could ambulate with or without the assistance of crutches. The time of ambulation without any assistance (unassisted ambulation) was the time considered on the data sheet.

All patients were interviewed by the anesthesiologist the day after surgery, to evaluate postoperative pain and adverse effects. The patient-recorded data were subsequently collected.

\section{Statistical analysis}

A power analysis considered a change of $30 \mathrm{~mm}$ in the VAS score as a significant clinical difference, a standard deviation (SD) of $35 \mathrm{~mm}$ was reported in previous studies, a type I error of $5 \%$ and a type II error of $10 \%$ yielded a sample size of at least 30 patients in each group.

The percentage of patients requiring rescue analgesia in the three groups was compared using the Fisher's exact test. Statistical significance was considered at $P<$ 0.05 . The analysis of variance with post hoc Scheffé test was used to compare the mean \pm SD of the VAS pain scores and other continuous data among the three groups. Additionally, to control the assumption of the inter- and intraindividual variability across the VAS observation and its influence on statistical decision making, we also performed a nonparametric analysis of the VAS scores, using nonlinear mixed effect modeling (NONMEM). The model parameters (VAS scores versus time) were estimated using NONMEM version VI (Globomax LLC, Hanover, MD). For the parameters, interindividual variability was modeled using a constant coefficient of variation model,

$$
\theta_{i}=\theta_{\mathrm{TV}} \cdot\left(1+\eta_{i}\right)
$$

where $\theta_{i}$ refers to the individual value of the parameter, $q_{\mathrm{TV}}$ is the typical value of the parameter, and $\eta$ is a normally distributed random variable with mean zero and a variance of $\omega^{2}$. Individual variability is reported as $\omega$, the SD of $\eta$ in the log domain, which is approximately the coefficient of variation in the standard domain. Residual intraindividual variability was modeled using a standard additive error model,

$$
\mathrm{DV}_{\mathrm{obs}}=\mathrm{DV}_{\mathrm{eps}}+\epsilon
$$

where $\mathrm{DV}_{\text {obs }}$ refers to the observed dependent variable, and $\mathrm{DV}_{\text {exp }}$ refers to the predicted dependent variable, $\epsilon$ is normally distributed with mean zero and variance $\sigma^{2}$. The objective function for the analysis was $-2 \log$ likelihood (-2LL). 


\begin{tabular}{lccc}
\hline & Group B $(n=30)$ & Group T $(n=30)$ & Group BT $(n=30)$ \\
\hline Age $(\mathrm{yr})$ & $34.0 \pm 11.0$ & $32.9 \pm 10.4$ & $36.6 \pm 11.6$ \\
Weight $(\mathrm{kg})$ & $69.6 \pm 7.1$ & $68.9 \pm 7.5$ & $71.5 \pm 7.7$ \\
Gender $(\mathrm{M} / \mathrm{F})$ & $17 / 13$ & $16 / 14$ & $15 / 15$ \\
Time of surgery $(\mathrm{min})$ & $40.5 \pm 7.1$ & $39.0 \pm 7.5$ & $41.5 \pm 6.9$ \\
\hline
\end{tabular}

$\mathrm{B}=$ bupivacaine; $\mathrm{T}=$ tramadol.

VAS group BUPIVAC

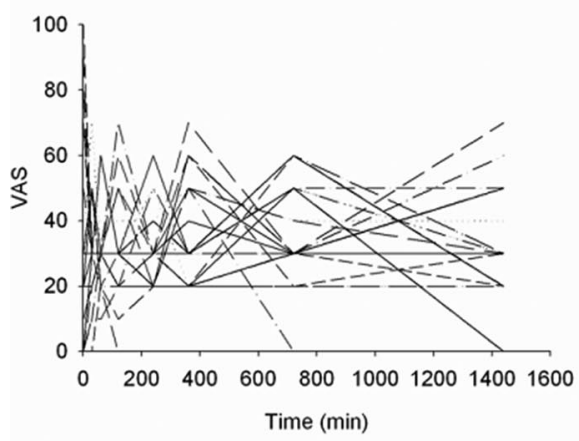

IPRED group BUPIVAC

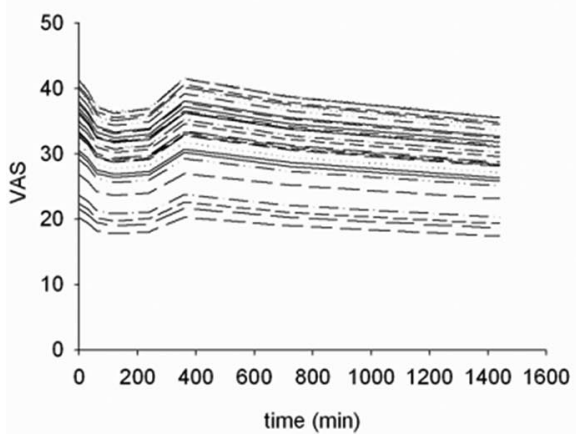

VAS group TRAMADOL

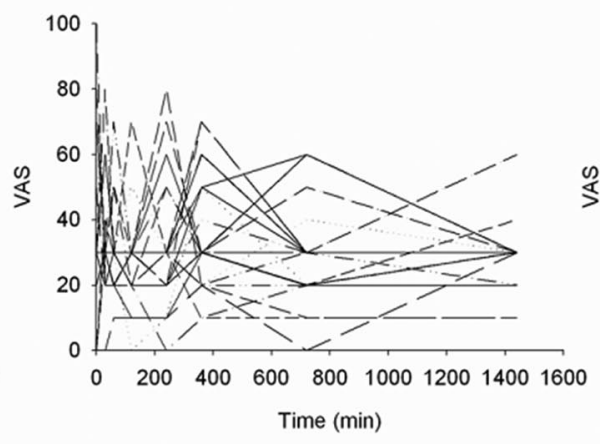

IPRED group TRAMADOL

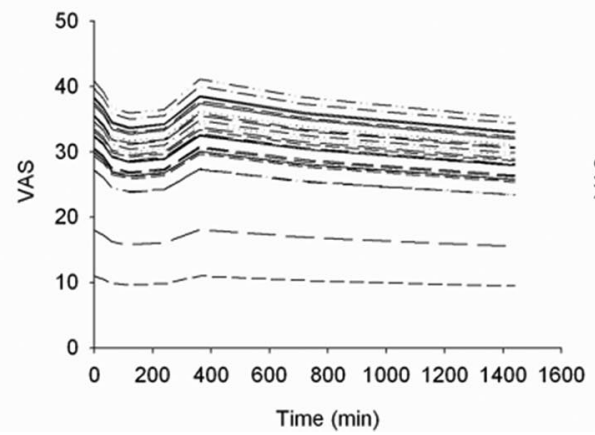

VAS group BUPIVAC + TRAMADOL

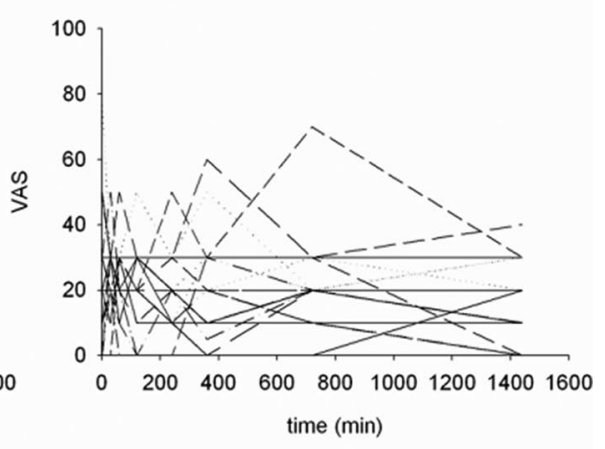

IPRED group BUPIVAC + TRAMADOL

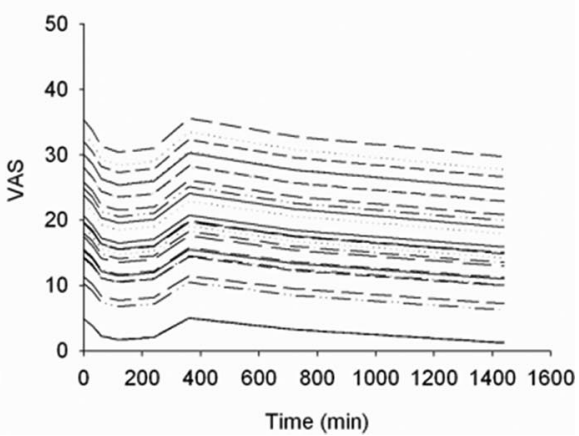

Figure 1. Patient visual analog scores (VAS) at rest: Upper part: Individual measured VAS scores versus time for the three groups. Lower part: Individual (post hoc) predicted VAS scores as predicted by NONMEM for each group.

The possible therapeutic effect of tramadol, bupivacaine, or the combination of both drugs on the VAS score was modeled as an additional effect. We first assumed that the underlying model which describes the relationship between the VAS versus time would not be influenced by the drugs used. Therefore, we first estimated the $-2 L L$ when no additional effect was present. Thereafter, we estimated the $-2 \mathrm{LL}$ adding an additional effect on one or more of the therapeutic groups. This additional effect was significantly compared with no effect if the difference in objective function with no effect was more than $P<0.05\left(\chi^{2}\right.$ test) or 3.84 difference in the $-2 \mathrm{LL}$ adding 1 parameter for nested models. Various models were tested, being group $\mathrm{B} \neq$ group $\mathrm{T}=$ group $\mathrm{BT}$; group $\mathrm{T} \neq$ group $\mathrm{B}=$ group $\mathrm{BT}$; group $\mathrm{B}=$ group $\mathrm{T} \neq$ group $\mathrm{BT}$; and group $\mathrm{B} \neq$ group $\mathrm{T} \neq$ group $\mathrm{BT}$. For the purpose of the analysis, we also assumed a similar additional effect on the VAS at all time points per group. The control file of the final best fitting model can be found in Appendix.

\section{RESULTS}

Demographic and surgical data are presented in Table 1 . There were no statistically significant differences among the three groups with respect to age, weight, gender, and duration of surgery.

The recorded postoperative VASr and VASm for each patient versus time are plotted in the upper part of Figures 1 and 2, respectively. At all times and for both VASr and VASm, the VAS scores were significantly lower in group BT when compared with groups B and T, as shown in Tables 2 and 3. The additional NONMEM analysis showed a similar result. The best model was found when an additional therapeutic effect was added to group BT, however, without differentiating a separate effect between groups $B$ and $T$. This final model resulted in a lower -2LL (difference of 39 points) and it was compared with the model without additional effect, which represents a large statistical significance $(P \ll$ 0.01 ) between group BT versus groups B and T. A model predicting a different effect among the three groups did 

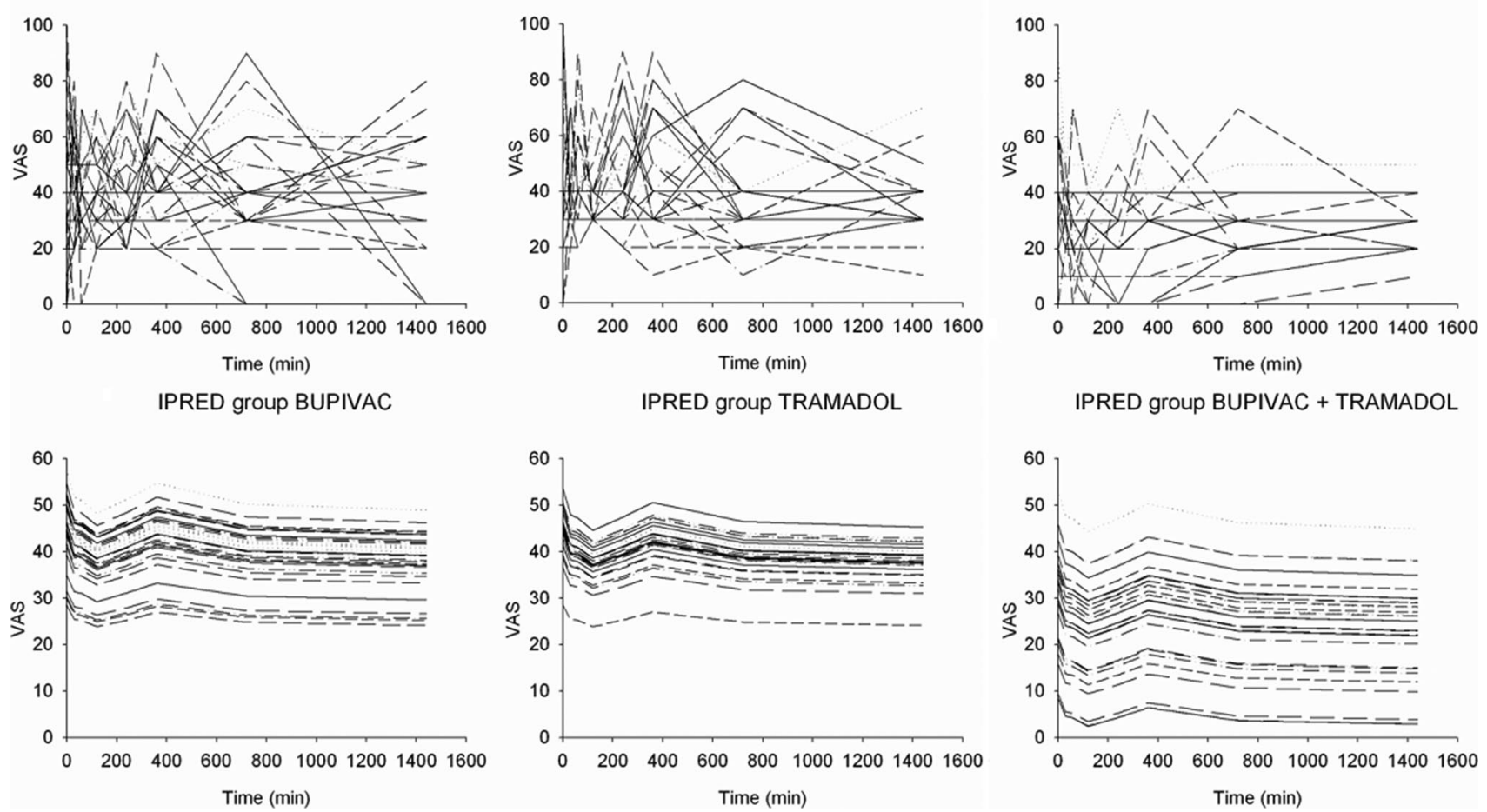

Figure 2. Patient visual analog scores (VAS) during mobilization: Upper part: Individual measured VAS scores versus time for the three groups. Lower part: Individual (post hoc) predicted VAS scores as predicted by NONMEM for each group.

Table 2. Visual Analog Scores at Rest (VASr) as Mean \pm SD at Different Time Intervals Following Surgeries

\begin{tabular}{lccccc}
\hline & $\begin{array}{c}\text { Group B } \\
(n=30)\end{array}$ & $\begin{array}{c}\text { Group T } \\
(n=30)\end{array}$ & $\begin{array}{c}\text { Group BT } \\
(n=30)\end{array}$ & $P(\mathrm{~B}+\mathrm{T}$ vs B $)$ & $P(\mathrm{~B}+\mathrm{T}$ vs T) \\
\hline VASr-0 min & $32 \pm 26$ & $31 \pm 23$ & $19 \pm 20$ & 0.044 & 0.042 \\
VASr-30 min & $31 \pm 13$ & $30 \pm 15$ & $18 \pm 13$ & 0.003 & 0.008 \\
VASr-60 min & $29 \pm 8$ & $28 \pm 13$ & $18 \pm 14$ & 0.0001 & 0.005 \\
VASr-2 h & $31 \pm 14$ & $28 \pm 12$ & $16 \pm 14$ & 0.0001 & 0.005 \\
VASr-4 h & $31 \pm 14$ & $30 \pm 18$ & $14 \pm 13$ & 0.0001 & 0.0001 \\
VASr-6 h & $34 \pm 16$ & $36 \pm 19$ & $16 \pm 17$ & 0.0001 & 0.001 \\
VASr-12 h & $35 \pm 16$ & $29 \pm 16$ & $15 \pm 15$ & 0.0001 & 0.0001 \\
VASr-24 h & $30 \pm 17$ & $29 \pm 12$ & $12 \pm 13$ & 0.0001 & \\
\hline
\end{tabular}

$\mathrm{B}=$ bupivacaine; $\mathrm{T}=$ tramadol.

Table 3. Visual Analog Scores During Motion (VASm) as Mean \pm SD at Different Time Intervals Following Surgeries

\begin{tabular}{lccccc}
\hline & $\begin{array}{c}\text { Group B } \\
(n=30)\end{array}$ & $\begin{array}{c}\text { Group T } \\
(n=30)\end{array}$ & $\begin{array}{c}\text { Group BT } \\
(n=30)\end{array}$ & $P(\mathrm{~B}+\mathrm{T}$ vs B) & $P(\mathrm{~B}+\mathrm{T}$ vs T) \\
\hline VASm-0 min & $44 \pm 26$ & $43 \pm 24$ & $30 \pm 22$ & 0.038 & 0.045 \\
VASm-30 min & $41 \pm 18$ & $37 \pm 11$ & $26 \pm 16$ & 0.003 & 0.01 \\
VASm-60 min & $37 \pm 16$ & $41 \pm 16$ & $24 \pm 18$ & 0.013 & 0.001 \\
VASm-2 h & $40 \pm 17$ & $36 \pm 10$ & $22 \pm 15$ & 0.0001 & 0.0001 \\
VASm-4 h & $40 \pm 19$ & $42 \pm 18$ & $23 \pm 17$ & 0.0001 & 0.0001 \\
VASm-6 h & $43 \pm 19$ & $45 \pm 21$ & $25 \pm 17$ & 0.002 & 0.0001 \\
VASm-12 h & $40 \pm 21$ & $38 \pm 16$ & $24 \pm 14$ & 0.024 & 0.002 \\
VASm-24 h & $36 \pm 22$ & $37 \pm 11$ & $26 \pm 11$ & & 0.0001 \\
\hline
\end{tabular}

$\mathrm{B}=$ bupivacaine; $\mathrm{T}=$ tramadol.

not result in a better model fit than the final model. The individual predicted VAS scores for each group at rest and during mobilization are shown in Figures 1 and 2D-F. The typical values for both conditions (rest and mobilization) at the various time points are given in Figures 3 and 4 . For both conditions, a highly significant difference is seen between the typical values for group
BT versus groups B and T. Groups B and T have similar typical values in the model. The interindividual variability analysis resulted in a coefficient of variation of $18 \%$. The beneficial effect of the combined therapy versus single therapy resulted in a typical VAS decrease of 14.3 with a coefficient of variation of $63 \%$. The additive residual intraindividual error was 15.3. 


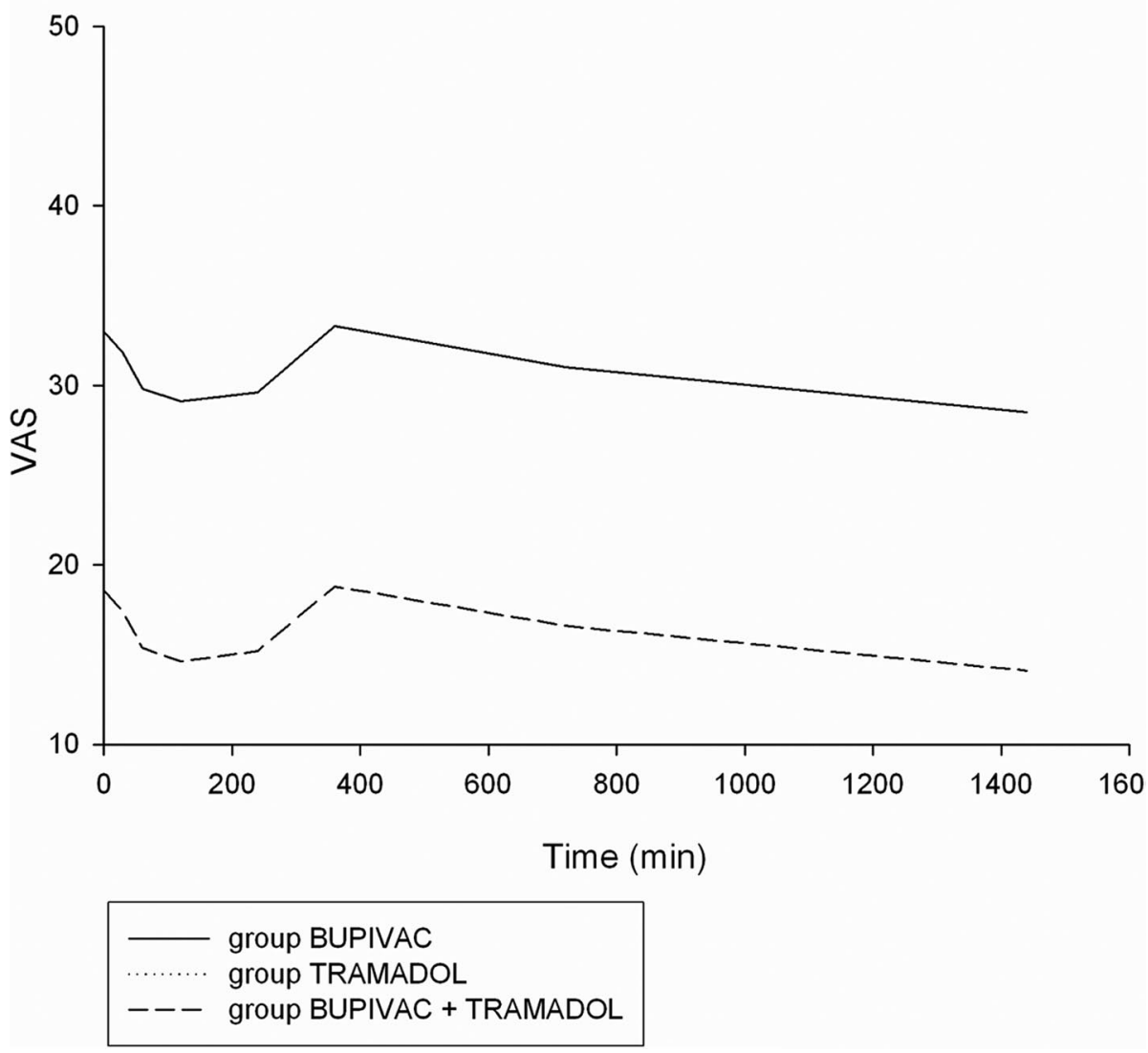

PRED move
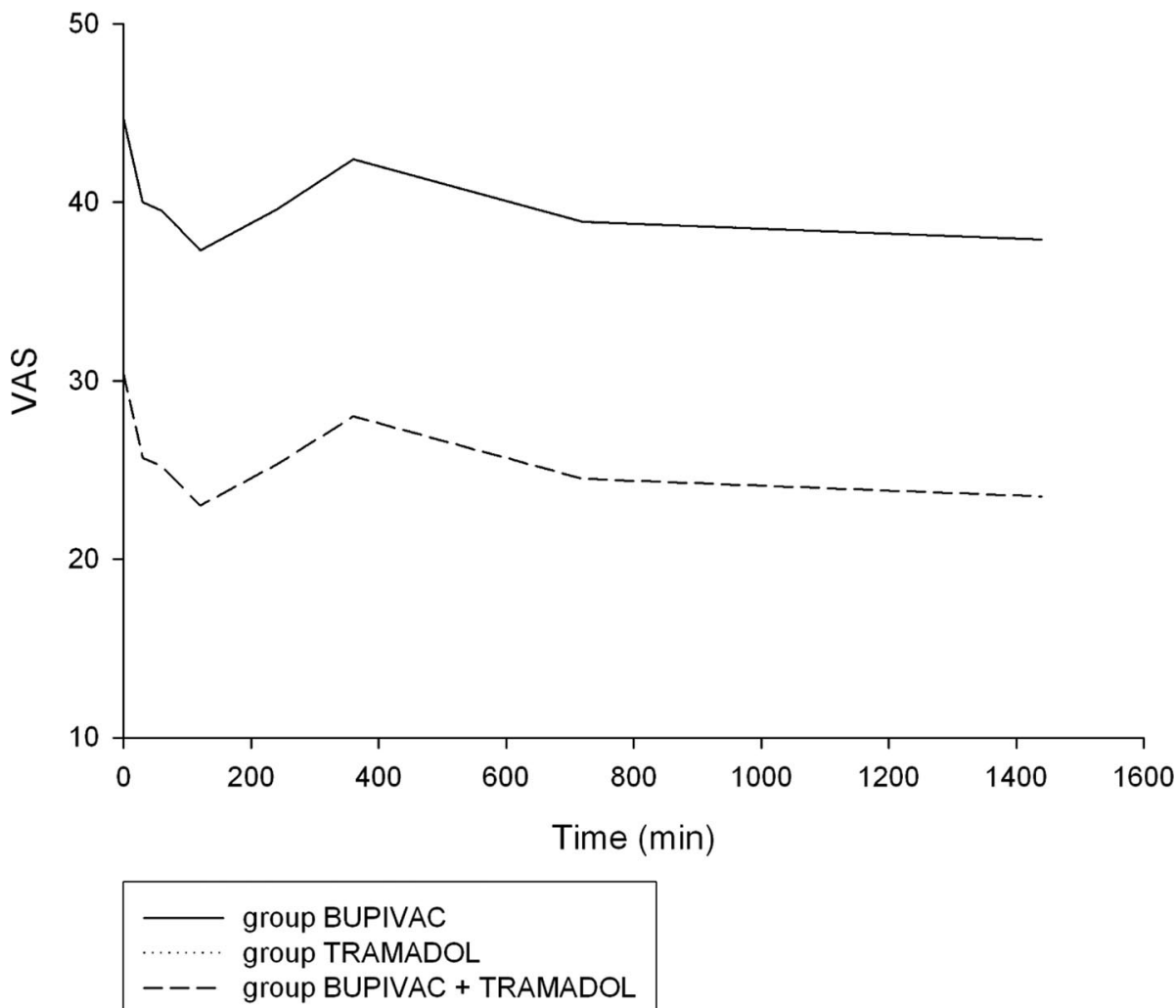

Figure 3. Typical population (post hoc) predicted visual analog scores (VAS) for groups B and T (solid line) and group BT (dotted line) at rest.
Figure 4. Typical population (post hoc) predicted visual analog scores (VAS) for groups B and T (solid line) and group BT (dotted line) during mobilization. 
Table 4. Postoperative Quality of Analgesia and Time for Discharge and Ambulation

\begin{tabular}{lccccc}
\hline & $\begin{array}{c}\text { Group B } \\
(n=30)\end{array}$ & $\begin{array}{c}\text { Group T } \\
(n=30)\end{array}$ & $\begin{array}{c}\text { Group BT } \\
(n=30)\end{array}$ & $P(\mathrm{~B}+$ T vs B) & $P(\mathrm{~B}+$ T vs T) \\
\hline $\begin{array}{l}\text { No. and \% of patients } \\
\quad \text { requiring rescue }\end{array}$ & $27(90 \%)$ & $26(86.7 \%)$ & $8(26.7 \%)$ & 0.0001 & 0.0001 \\
$\quad$ analgesia (Paracetamol) & & & & & \\
No. of rescue analgesia & $1.7 \pm 1.0$ & $1.4 \pm 0.8$ & $0.4 \pm 0.6$ & 0.001 & 0.001 \\
Total dose (no. of tablets/24 h) & $3.3 \pm 1.9$ & $2.7 \pm 1.6$ & $0.8 \pm 1.3$ & 0.001 & 0.001 \\
Time to discharge (h) & $7.5 \pm 2.7$ & $7.1 \pm 2.4$ & $5.0 \pm 1.8$ & 0.001 & 0.004 \\
Time to ambulation (h) & $9.0 \pm 4.3$ & $9.2 \pm 5.7$ & $4.7 \pm 2.1$ & 0.001 & 0.001 \\
\hline
\end{tabular}

No. $=$ number; $\%=$ percentage; $\mathrm{B}=$ bupivacaine; $\mathrm{T}=$ tramadol.

The percentage of patients in group BT requiring rescue analgesia was $26.7 \%$, which is significantly less than the percentage of patients in either group B $(90 \%)$ or group $\mathrm{T}(86.7 \%)$. Also, the numbers of rescue analgesia requests as well as the cumulative $24 \mathrm{~h}$ analgesic consumption were significantly smaller in group BT compared with groups B or T (Table 4). Subsequently, time to discharge and time to unassisted ambulation were significantly shorter in group BT than in groups B or T (Table 4).

No patient, in any group, showed and/or recorded postoperative headache, nausea, vomiting, dizziness, or somnolence.

\section{DISCUSSION}

In the present study, we found lower VAS pain scores, a longer duration of analgesia, and a decrease in the $24 \mathrm{~h}$ consumption of rescue analgesia in the group receiving the IA combination of $100 \mathrm{mg}$ tramadol and $0.25 \%$ bupivacaine when compared with the groups receiving bupivacaine or tramadol injection alone. There was also earlier recovery of unassisted ambulation and home discharge for the combination group. No side effects were detected in any groups.

Different adjuvant drugs, including opioids, nonsteroidal antiinflammatory drugs, ketamine, clonidine, and neostigmine, have been added to IA LAs to improve the duration and quality of analgesia after knee arthroscopy. A comparative study showed that the most effective drugs administered IA are neostigmine and clonidine when compared with tenoxicam, morphine, and bupivacaine. ${ }^{1}$ Evidence indicates that a variety of these drugs have synergistic effects through a local, rather than a central, mechanism.

IA tramadol has also been used for pain management of these patients. ${ }^{5}$ Alagol et al..$^{5}$ showed that tramadol $100 \mathrm{mg}$ without LA provided lower VAS pain scores and longer analgesic effect after IA administration more than after IV injection of the same doses with no significant side effects.

In our study, IA tramadol had an analgesic effect similar to that of IA bupivacaine. It is possible that the combination of IA tramadol and LA provides its regional analgesic effect by a multimodal mechanism of action, which gives a synergistic effect, as evidenced by the decreased VAS pain scores, decreased need for postoperative analgesics, and an increased analgesic duration, as well as the early unassisted ambulation and discharge.

Although tramadol was initially considered to be a weak $\mu$-opioid agonist, it appears to have multimodal mechanisms of action. It is now accepted that, in addition to the $\mu$-opioid agonist effect, tramadol enhances the function of the spinal descending inhibitory pathway by inhibition of reuptake of both 5-hydroxytryptamine (5-HT) and norepinephrine, together with presynaptic stimulation of 5-HT release. ${ }^{6,7}$

The LA action of tramadol remains unproven. 5-HT3 receptors are expressed on the peripheral and spinal terminals of the nociceptive primary afferent fibers, as well as on the superficial lamina of the dorsal horn, which indicates possible peripheral sites of analgesic action for tramadol. ${ }^{8,9}$ Mert et al. ${ }^{10}$ have shown a definitive LA effect of tramadol in experiments on frog sciatic nerves. In their animal study, the nerve conduction block of tramadol was 3-6 times weaker than that of lidocaine. Although lidocaine inhibits $\mathrm{Na}$ channels, it has been suggested that tramadol inhibits $\mathrm{K}$ channels.

Although the analgesic effect of IA opioid with or without LA after arthroscopy is controversial, the existence of agonist-specific IA opioid receptors has been well documented in recent years. Boden et al. ${ }^{11}$ found a significant analgesic effect of IA opioid when injected alone and a synergistic effect when added to the LA. It was postulated that peripheral opioid receptors may be activated only in the presence of tissue inflammation. The timing of IA opioid administration may also be an important factor. Whitford et al. ${ }^{12}$ found that maintaining tourniquet inflation for 10 min after IA opioid injection improved postoperative analgesia, presumably by allowing tissue binding before tourniquet release, and the subsequent posttourniquet hyperemia and tissue washout.

Furthermore, several publications reported that tramadol, when added to LA, modifies peripheral anesthesia. ${ }^{13,14}$ Kapral et al. ${ }^{13}$ reported that tramadol increased the duration of analgesia when added to mepivacaine for axillary plexus blockade.

For ethical reasons, we did not include a control group receiving IA placebo and parenteral tramadol, since it has been reported that tramadol provided a 
longer analgesic effect after IA administration than after IV injection of the same doses. ${ }^{5}$

Headache, nausea, vomiting, dizziness, and somnolence had been major side effects of IV tramadol when used for postoperative analgesia. ${ }^{15}$ The incidence of nausea and vomiting seems to be related mainly to the peak serum concentrations reached by a direct IV loading dose, which causes more symptoms than a subsequent infusion or local infiltration. ${ }^{16,17}$ This may partially explain the absence of side effects after the IA tramadol administration in our patients.

In conclusion, our report showed that the IA admixture of $100 \mathrm{mg}$ tramadol with $0.25 \%$ bupivacaine decreased both VASr and VASm and provided longer postoperative analgesia than that produced by IA injection of either bupivacaine or tramadol alone. This was also associated with earlier recovery of unassisted ambulation and home discharge. Also, the IA combination of tramadol-bupivacaine was not associated with any side effects.

\section{APPENDIX}

\$PROB Intraartic_tramadol_Bupivac

\$DATA DATA_REST.txt

\$INPUT ID TIME POD VAS=DV TRT

\$PRED

IF (POD.EQ.0) THEN

$\mathrm{TY}=\operatorname{THETA}(1)^{*}(1+\operatorname{ETA}(1))$

ENDIF

IF (POD.EQ.30) THEN

$\mathrm{TY}=\operatorname{THETA}(2)^{*}(1+\mathrm{ETA}(1))$

ENDIF

IF (POD.EQ.60) THEN

$\mathrm{TY}=\mathrm{THETA}(3)^{*}(1+\mathrm{ETA}(1))$

ENDIF

IF (POD.EQ.120) THEN

$\mathrm{TY}=\operatorname{THETA}(4)^{*}(1+\mathrm{ETA}(1))$

ENDIF

IF (POD.EQ.240) THEN

$\mathrm{TY}=\mathrm{THETA}(5)^{*}(1+\mathrm{ETA}(1))$

ENDIF

IF (POD.EQ.360) THEN

$\mathrm{TY}=\operatorname{THETA}(6)^{*}(1+\mathrm{ETA}(1))$

ENDIF

IF (POD.EQ.720) THEN

$\mathrm{TY}=\mathrm{THETA}(7)^{*}(1+\mathrm{ETA}(1))$

ENDIF

IF (POD.EQ.1440) THEN

$\mathrm{TY}=\operatorname{THETA}(8)^{*}(1+\mathrm{ETA}(1))$

ENDIF

IF (TRT.EQ.1) THEN

LESS $=0$

ENDIF

IF (TRT.EQ.2) THEN

LESS $=0$

ENDIF

IF (TRT.EQ.3) THEN

$\operatorname{LESS}=\operatorname{THETA}(9)^{*}(1+\operatorname{ETA}(2))$
ENDIF

IPRED $=$ TY-LESS

$Y=\operatorname{IPRED}+\operatorname{EPS}(1)$

\$THETA

$(0,20,100)$; Theta 01

$(0,32,100)$; Theta 02

$(0,26,100)$; Theta 03

$(0,23,100)$; Theta 04

$(0,19,100)$; Theta 05

$(0,7,100)$; Theta 06

$(0,20,100)$; Theta 07

$(0,20,100)$; Theta 08

-0.1 ; Theta 9

\$OMEGA 0.01 0.01; Between subject variability

\$SIGMA 50; Residual variability

$\$ E S T I M A T I O N$ MAX $=1000$ PRINT $=1$ NOABORT

$\mathrm{METHOD}=1 \mathrm{SIG}=3$

\$TABLE ID TIME POD VAS TRT IPRED

\section{ACKNOWLEDGMENT}

We thank Mrs. Ghina Kassem for reviewing English.

\section{REFERENCES}

1. Alagol A, Calpur OU, Usar PS, Turan N, Pamukcu Z. Intraarticular analgesia after arthroscopic knee surgery: comparison of neostigmine, clonidine, tenoxicam, morphine and bupivacaine. Knee Surg Sports Traumatol Arthrosc 2005;13:658-63

2. Raja SN, Dickstein RE, Johnson CA. Comparison of postoperative analgesic effects of intraarticular bupivacaine and morphine following arthroscopic knee surgery. Anesthesiology 1992;77:1143-7

3. Cook TM, Tuckey JP, Nolan JP. Analgesia after day-case knee arthroscopy: double-blind study of intra-articular tenoxicam, intraarticular bupivacaine and placebo. Br J Anaesth 1997;78:163-8

4. Batra YK, Mahajan R, Bangalia SK, Nagi ON, Dhillon MS. Bupivacaine/ketamine is superior to intra-articular ketamine analgesia following arthroscopic knee surgery. Can J Anaesth 2005;52:832-6

5. Alagol A, Calpur OU, Kaya G, Pamukcu Z, Turan FN. The use of intraarticular tramadol for postoperative analgesia after arthroscopic knee surgery: a comparison of different intraarticular and intravenous doses. Knee Surg Sports Traumatol Arthrosc 2004; $12: 184-8$

6. Raffa RB, Friderich E, Reimann W, Shank RP, Codd EE, Vaught JL. Opioid and nonopioid components independently contribute to the mechanism of action of tramadol, an atypical opioid analgesic. J Pharmacol Exp Ther 1992;260:275-85

7. Bamigbade TA, Davidson C, Langford RM, Stamford JA. Actions of tramadol, its enantiomers and principal metabolite, O-desmethyltramadol, on serotinine (5-HT) efflux and uptake in the rat dorsal raphe nucleus. Br J Anaesth 1997;79:352-6

8. Kayser V, Besson JM, Guilbaud G. Evidence for noradrenergic component in the antinociceptive effect of the analgesic agenttramadol in an animal model of clinical pain, the arthritic rat. Eur J Pharmacol 1992;224:83-8

9. Arcioni R, della Rocca M, Romano S, Romano R, Pietropaoli P, Gasparetto A. Ondansetron inhibits the analgesic effects of tramadol: a possible 5-HT(3) spinal receptor involvement in acute pain in humans. Anesth Analg 2002;94:1553-7

10. Mert T, Gunes Y, Guven M, Gunay I, Gocmen C. Differential effects of lidocaine and tramadol on modified nerve impulse by 4-aminopyridine in rats. Pharmacology 2003;69:68-73

11. Boden BP, Fassler S, Cooper S, Marchetto PA, Moyer RA. Analgesic effect of intraarticular morphine, bupivacaine, and morphine/bupivacaine after arthroscopic knee surgery. Arthroscopy 1994;10:104-7

12. Whitford A, Healy M, Joshi GP, McCarroll SM, O’Brien TM. The effect of tourniquet release time on the analgesic efficacy of intraarticular morphine after arthroscopic knee surgery. Anesth Analg 1997;84:791-3 
13. Kapral S, Gollmann G, Waltl B, Likar R, Sladen RN, Weinstabl C, Lehofer F. Tramadol added to mepivacaine prolongs the duration of an axillary brachial plexus blockade. Anesth Analg 1999;88:853-6

14. Robaux S, Blunt C, Viel E, Cuvillon P, Nouguier P, Dautel G, Boileau S, Girard F, Bouaziz H. Tramadol added to $1.5 \%$ mepivacaine for axillary brachial plexus block improves postoperative analgesia dose-dependently. Anesth Analg 2004; 98:1172-7
15. Shipton EA. Tramadol: present and future. Anesth Intensive Care 2000;28:363-74

16. Radbruch L, Grond S, Lehmann KA. A risk-benefit assessment of tramadol in the management of pain. Drug Saf 1996;15:8-29

17. Lintz W, Beier H, Gerloff J. Bioavailability of tramadol after i.m. injection in Comparison to i.v. infusion. Int J Clin Pharmacol Ther 1999;37:175-83

\section{ERRATUM}

In the article "Electrical Nerve Stimulation or Ultrasound Guidance for Lateral Sagittal Infraclavicular Blocks: A Randomized, Controlled, Observer-Blinded, Comparative Study" which appeared in the June 2008 issue of volume 106 of Anesthesia \& Analgesia on pages 1910-5, there was as a copyediting error concerning the needle position of ultrasound guided blocks (Methods, page 1911, first column, last line). It is stated that "the needle was placed in 9 o'clock position." It should be "the needle was placed in 8 o'clock position."

This error has been corrected in the online version of the article available at www.anesthesia-analgesia.org.

1. Sauter AR, Dodgson MS, Stubhaug A, Halstensen AM, Klaastad O. Electrical nerve stimulation or ultrasound guidance for lateral sagittal infraclavicular blocks: a randomized, controlled, observer-blinded, comparative study. Anesth Analg 2008;106:1910-5 